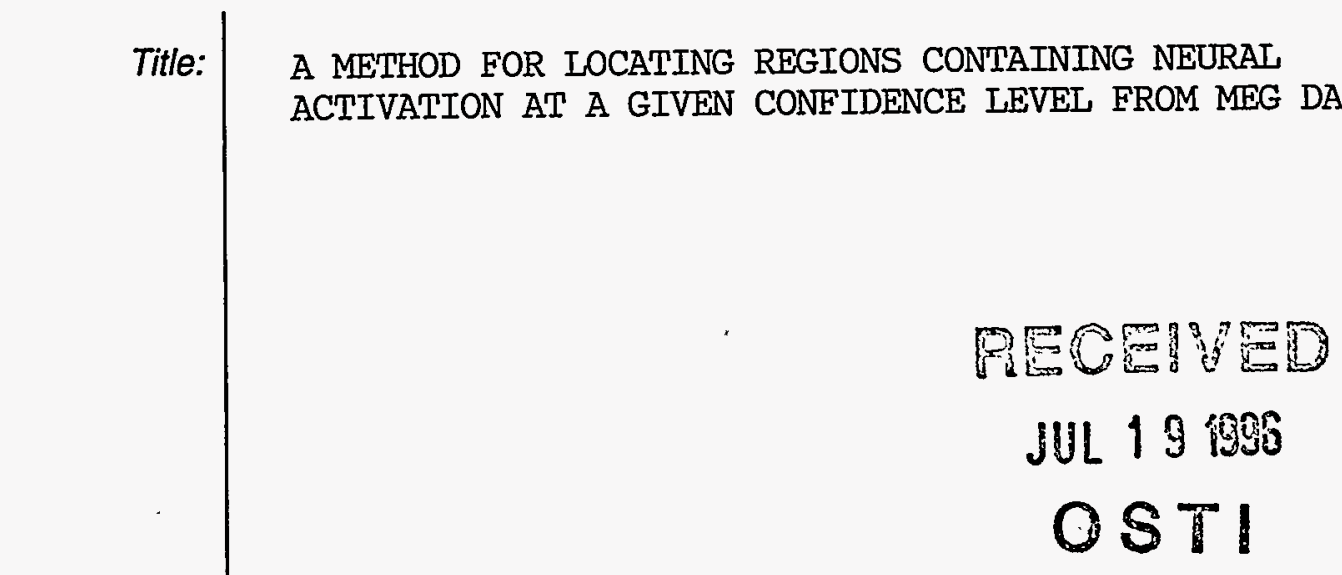

Author(s): $\quad$ D. M. Schmidt, J. S. George

Submitted to: Biomagnetism 196, Santa Fe, NM, February 1996

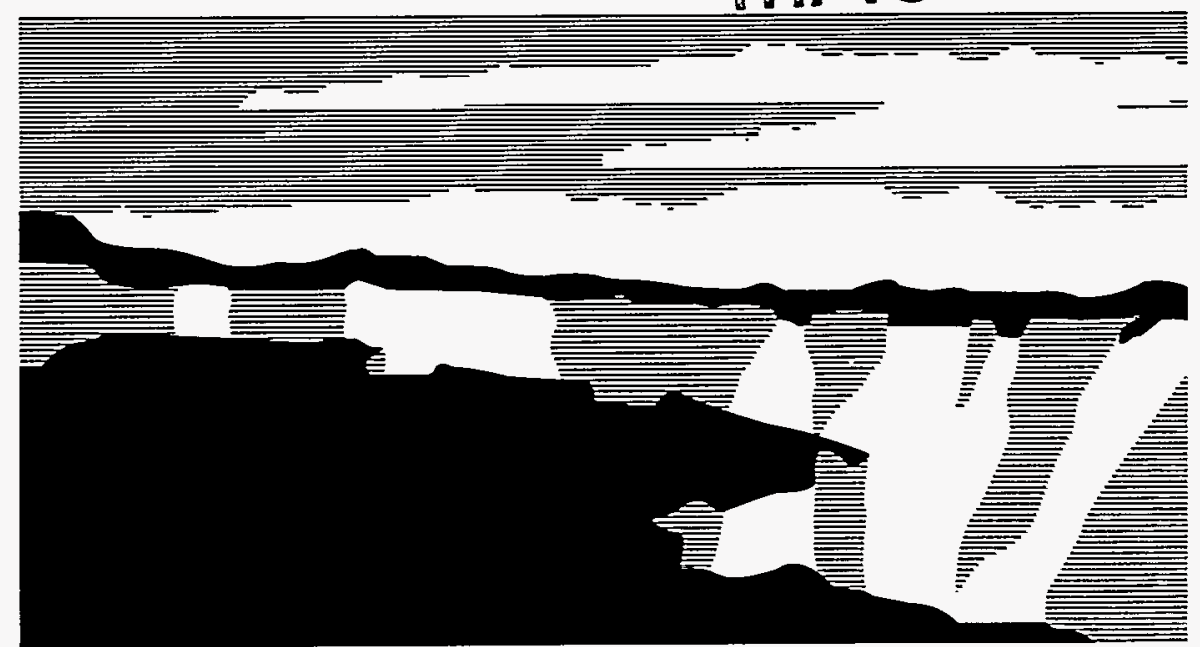

Los Alamos National Laboratory, an affirmative action/equal opportunity employer, is operated by the University of California for the U.S. Department of Energy under contract W-7405-ENG-36. By acceptance of this article, the publisher recognizes that the U.S. Government retains a nonexclusive, royalty-free license to publish or reproduce the published form of this contribution, or to allow others to do so, for U.S. Government purposes. The Los Alamos National Laboratory requests that the publisher identify this article as work performed under the auspices of the U.S. Department of Energy. 


\section{DISCLAIMER}

This report was prepared as an account of work sponsored by an agency of the United States Government. Neither the United States Government nor any agency thereof, nor any of their employees, makes any warranty, express or implied, or assumes any legal liability or responsibility for the accuracy, completeness, or usefulness of any information, apparatus, product, or process disclosed, or represents that its use would not infringe privately owned rights. Reference herein to any specific commercial product, process, or service by trade name, trademark, manufacturer, or otherwise does not necessarily constitute or imply its endorsement, recommendation, or favoring by the United States Government or any agency thereof. The views and opinions of authors expressed herein do not necessarily state or reflect those of the United States Government or any agency thereof. 


\title{
A Method for Locating Regions Containing Neural Activation at a Given Confidence Level from MEG Data
}

\author{
Schmidt, D.M., George, J.S. \\ Biophysics Group, Los Alamos National Laboratory, Los Alamos, New Mexico, USA
}

\section{Introduction}

The MEG inverse problem does not have a general, unique solution. Unless restrictive model assumptions are made, there are generally many more free parameters than measurements and there exist "silent sources"-current distributions which produce no external magnetic field. By weighting solutions according to how well each fits our prior notion about what properties good solutions should have, it may be possible to obtain a single current distribution that "best fits" the data and our expectations. However, in general there will still exist a number of different current distributions which fit both the data and our prior expectations sufficiently well. For example, a simulated data set based on a single or several dipoles can generally be fit equally well by a distributed current minimum-norm reconstruction. In experimental data it is often possible to find a relatively small number of dipoles which both fit the data and have a norm not much larger than that of the minimum-norm solution. Moreover, the few-dipole solutions often have currents in different regions than the corresponding minimum-norm solution. Because there exist well-fitting current distributions which may have current in significantly different locations, it can be misleading to infer locations of stimulus-correlated neural activity based on a single, best-fitting current distribution. We demonstrate here a method for inferring the location and number of regions containing neural activation by considering all possible current distributions within a given model (not just the most likely one) weighted according to how well each fits both the data and our prior expectations.

\section{Methods}

We employ a Bayesian approach which considers a posterior probability distribution, $P(j \mid$ data $)$, that assigns each current distribution, $j$, a relative likelihood based on how well each distribution fits the data and meets one's prior expectation-explicitly defined by a prior probability distribution, $P(j)$. Specifically, Bayes theorem[1] states that

$$
P(j \mid \text { data }) \propto P(\text { data } \mid j) P(j)
$$

where $P($ data $\mid j)$ is the likelihood of the data given the current distribution, $j$. In some over-determined problems the information from the data dominates, and the posterior probability distribution is highly peaked. In such cases inferences from the most likely solution may be reliable. However, with MEG data the posterior distribution is generally quite broad because of the ill-posed nature of the problem and because there are often more free parameters than measurements. Most methods widely used for analysis of MEG data infer neural activity based on a single best-fitting current distribution within the context of a given source model. Implicit model assumptions strongly influence the nature of the reconstructed current distribution but the consequences of model assumptions are seldom considered. In our Bayesian approach, model assumptions are made explicit in the form of the prior probability distribution. In addition, we infer activation from the full posterior distribution, which we believe will yield different and more reliable estimates than inferences made from a single "most likely" current distribution.

In our model, we assume that there are a small number of compact regions that contain the stimulus-correlated neural activity. This is appropriate, for example, for stimuli designed to generate a limited, focal evoked response. What we want to determine is how many of these regions are needed, $n$, and where they are located, $w$. Following Bayes theorem,

$$
P(n, w \mid \text { data }) \propto P(\text { data } \mid n, w) P(n, w)
$$

where $P(n, w \mid$ data $)$ is the posterior probability of the number and location of regions containing activity given the data, $P(n, w)$ is the prior probability for the number and location of these regions, and $P(\operatorname{data} \mid n, w)$ is the likelihood of the data given a particular number and location of regions. The data, however, do not depend directly on the number 
and location of regions; rather they depend on the neural current distribution. Thus, we need to sum over all possible current distributions consistent with a given number and location of activation regions:

$$
P(\text { data } \mid n, w)=\sum_{j} P(\text { data } \mid j) P(j \mid n, w)
$$

where $P(j \mid n, w)$ is the prior probability for a current distribution, $j$, given the number and location of the regions of activation, $n$ and $w$. Putting this together we have:

$$
P(n, w \mid \text { data }) \propto \sum_{j} P(\text { data } \mid j) P(j \mid n, w) P(n, w) .
$$

What we have essentially done is to change the problem from finding the best current distribution, which generally has a large number of parameters, to finding the best set of small regions, which has a much smaller number of parameters yet retains a rich set of possibilities within current space. To do this, we sum over all possible current distributions-a process known as marginalization.

In principle, determining confidence intervals within the Bayesian formalism is straight forward because all relevant information is contained in the posterior distribution. The $90 \%$ confidence interval for $\{n, w\}$, for example, is simply the set, $\{n, w\}_{90}$, for which the posterior, $P(n, w \mid$ data $)$, is greater than the value of the posterior for any other set of $\{n, w\}$ and the integral of the posterior over $\{n, w\}_{90}$ is $90 \%$ that of the integral over the full range of $\{n, w\}$. In practice we determined the confidence region by sampling the posterior using a Monte Carlo integration technique and constructing a histogram similar to that shown in Fig. 1. The integral of the posterior over the full range of $\{n, w\}$ is approximated by summing the product of the average value of the posterior at each histogram bin and the frequency of occurrence for that bin. To determine the $90 \%$ confidence interval we simply found the cut-off value of the posterior probability along the horizontal axis, $\alpha$, such that the integral of the posterior from

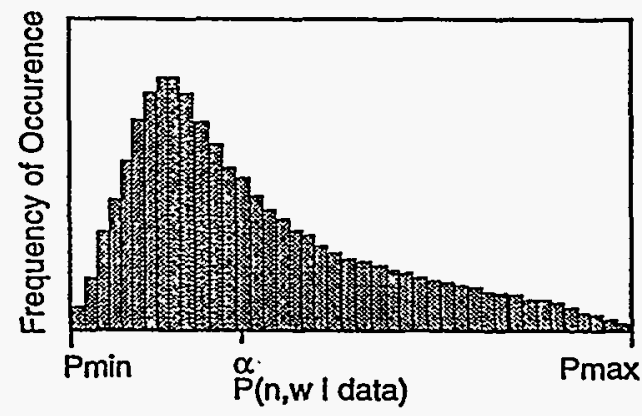

Fig. 1, Illustration of a histogram of values sampled from the posterior distribution, $P(n, w \mid$ data $)$. this value to the maximum value is $90 \%$ of the value of the integral over the whole range. The $90 \%$ confidence interval region consists of all values of $\{n, w\}$ for which $P(n, w \mid$ data $)$ is greater than or equal to this cut-off value.

\section{Results}

The use of this algorithm is demonstrated with simulated data consisting of a range of numbers and locations of current dipole sources. In order to be as realistic as possible we used a sensor configuration from a real experiment in which a visual evoked response was studied. In this experiment there were two placements of a 37 channel first order gradiometer array (BTI) around the back of the subject's head. The Sarvas model[2] was used to calculate the output from the 74 sensors for a given set of current dipoles. Gaussian noise with standard deviation $1 \mathrm{fT}$ was added to the simulated data which resulted in a noise level that was between $1 \%$ and $10 \%$ that of average signal strength, depending on the locations of the sources. The source volume consisted of 17,342 cortical volume elements (voxels) from the subject's anatomical MRI scan with the cortex segmented using tools developed in our laboratory and described elsewhere. $[3,4]$

For the compact regions of activation which make up our model we used spheres of $1 \mathrm{~cm}$ radius such that any voxel within a sphere is considered to be part of the "active" region. We allowed no more than 4 regions with relative prior probability of $1 ., 1 ., 1 ., 1 ., 7$ for 0 through 4 regions respectively. This specifies $P(n, w)$. For the prior probability of the current we used

$$
P(j \mid n, w) \propto \operatorname{Exp}\left(-\frac{1}{2} \sum_{i} \frac{\vec{j}_{i}^{2}}{V}\right)
$$

where $i$ enumerates all voxels within the regions specified by $\{n, w\}$, and $\vec{j}_{i}$ is the current dipole for voxel $i$. The parameter $V$ represents the value above which the squared current dipole moment for each voxel starts to become 
significantly improbable. This prior tends to exclude large currents. We used $V=500 n A^{2} m^{2}$, which is fairly large since the sources used in our simulation all had a strength of $10 \mathrm{nAm}$. Because Gaussian noise was added to the data, the likelihood of the data given a current distribution, $j$, is itself a Gaussian distribution:

$$
P(\text { data } \mid j) \propto E x p-\frac{1}{2} \sum_{k}\left(\frac{d_{k}-\sum_{i} \vec{A}_{k, i} \cdot \vec{j}_{i}}{\sigma_{k}}\right)^{2}
$$

where $d_{k}$ is the data value for channel $k, \sigma_{k}$ is the noise standard deviation for channel $k$, and $\vec{A}_{k, i}$ is the forward field value for channel $k$ with a unit dipole at voxel $i$.
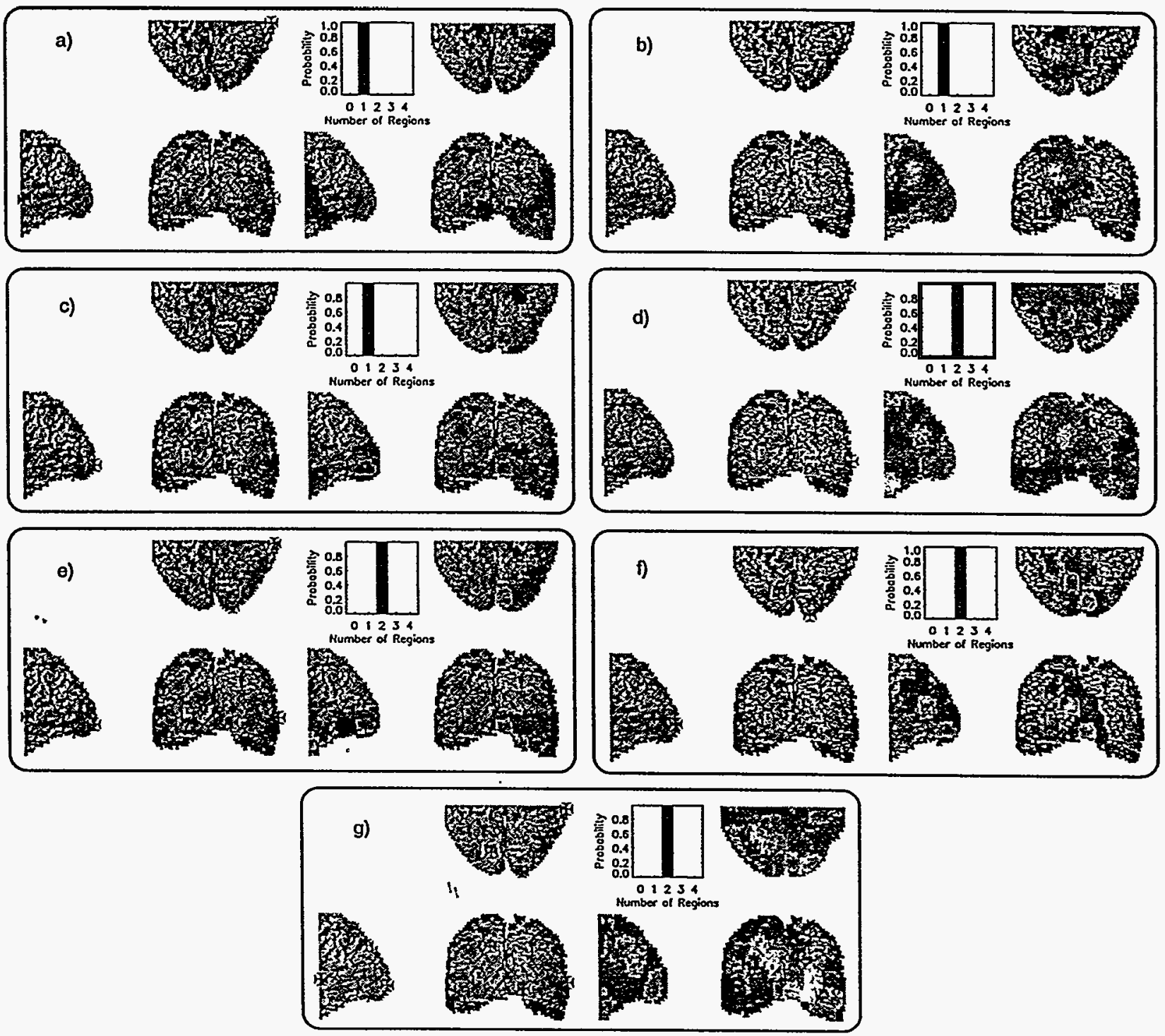

Fig. 2, Results of our method with simulated data. The location and number of true sources are shown in the left 3 cortical views within each panel. The $99.9 \%$ confidence intervals are shown as smooth grey patches on the right 3 views within each panel. Also shown is the posterior probability for the number of regions needed.

We evaluated the posterior by first randomly selecting values for the number and the locations of regions, $\{n, w\}$. For each set of $\{n, w\}$ we evaluated the posterior, $P(n, w \mid$ data $)$, by integrating over all current values, $\vec{j}_{i}$, for voxels within the selected regions of activation. This integral was evaluated semi-analytically, using the fact that the current is quadratic in the exponential; the computation was therefore relatively fast. The value of the posterior was 
recorded in a histogram. In addition, the maximum posterior value in which a voxel is active was recorded for each voxel. After sufficiently sampling $\{n, w\}$, the histogram of posterior values was examined to determine the cut-off value for the desired confidence level. All voxels whose maximum posterior value was greater than or equal to the cut-off value were assigned to the confidence level region. In addition, we integrated (or marginalized) over location, $w$, in order to determine the posterior probability for $n$, the number of regions needed.

The results are shown in Fig. 2. Each panel represents a different source configuration, with the total number of sources ranging from one to three. Within each panel the left three cortical orthogonal renderings show the projection of the location and number of the true sources, marked with a black symbol. A maximum intensity projection of the $99.9 \%$ confidence region needed to contain the activity is superimposed on the right three cortical renderings within each panel. The confidence regions appear as smooth grey-scale patches on top of the textured cortical surface. The shade of grey indicates relative likelihood within the confidence region (white being most likely). In addition, the marginal posterior probability for the number of regions, $n$, is shown in the bar chart within each panel.

In most cases the true number of regions is reconstructed and the true location of activity is within the confidence region. This region is not guaranteed to contain activity, but rather to probably contain activity at the given confidence level. Nevertheless, this is much better than the level of accuracy and confidence obtained with just the most likely solution - given by the lightest patches in the images.

\section{Discussion}

We have demonstrated a Bayesian approach for inferring the number and location of regions of neural activation from MEG data. Our model consists of a small number of small size regions and is not parameterized by a given current distribution. Rather, we marginalize over the rich set of current distributions consistent with our model in order to determine the relative likelihood for the location and number of regions of activation. This is different from an equivalent current dipole approach, for example, which severely restricts the set of possible current distributions in order to obtain a more well-behaved, over-determined problem. The equivalent current dipole solution is merely representative of the actual neural current and it is left to the experimenter to guess at where the actual locations of activation are. Furthermore, we use the Bayesian formalism to solve the "model order" problem by constructing a prior probability consisting of all possible numbers of regions we care to examine, with relative weights for each. Marginalization of the posterior probability then gives the relative likelihood of the number of regions needed from the information contained in the data. This is a promising and flexible approach. Future work will examine the effects of different sizes and numbers of regions, and will incorporate information about temporal as well as spatial aspects of the data for assessment of conditional probabilities.

\section{References}

[1] Bayes, T., An essay towards solving a problem in the doctrine of chances, Biometrika, 1958 (Reprint of 1763), 45: 293-315.

[2] Sarvas, J., Basic mathematical and electromagnetic concepts of the biomagnetic inverse problem, Phys. Med. Biol., 1987, 32: 11-22.

[3] Ranken, D.M., and George, J.S., MRIVIEW: An interactive computational tool for investigation of brain structure and function, Visualization '93, IEEE Computer Society, 1993: 324-331.

[4] George, J.S., Aine, C.J., Mosher, J.C., Schmidt, D.M., Ranken, D.M., Schlitt, H.A., Wood, C.C., Lewine, J.A., Belliveau, J.W., Mapping function in the human brain with MEG, anatomical MRI and functional MRI, J. Clin. Neurophysiology, 1995, 12: 406-431.

\section{Acknowledgments}

This work was supported in part by the National Institutes of Health, grant number DA/MH09972-01. 